\title{
Effect of poly(3,4-ethylenedioxythiophene) (PEDOT) in carbon-based composite electrodes for electrochemical supercapacitors
}

\author{
Chunhong Lei, Peter Wilson, Constantina Lekakou* \\ Division of Mechanical, Medical, and Aerospace Engineering, Faculty of Engineering and \\ Physical Sciences, University of Surrey, Guildford GU2 7XH, UK
}

Fax: +44 (0) 148368 6291;

Tel: +44 (0) 1483689622 ;

*E-mail: C.Lekakou@ @urrey.ac.uk

\begin{abstract}
\end{abstract}
Electrochemical double layer supercapacitor cells were fabricated and tested using composite electrodes of activated carbon with carbon black and poly(3,4-ethylenedioxythiophene) (PEDOT), and an organic electrolyte $1 \mathrm{M}$ $\mathrm{TEABF}_{4} / \mathrm{PC}$ solution. The effect of PEDOT on the performance of the EDLC cells was explored and the cells were characterised by electrochemical impedance spectroscopy (EIS), cyclic voltammetry and galvanostatic charge-discharge. A generalised 
equivalent circuit model was developed for which numerical simulations were performed to determine the properties and parameters of its components from the EIS data. It was found that the proposed model fitted successfully the data of all tested cells. PEDOT enhanced the electrode and cell capacitance via its pseudo-capacitance effect up to a maximum value for an optimum PEDOT loading and greatly increased the energy density of the cell while the maximum power density has been still maintained at supercapacitor levels. Furthermore, PEDOT replaced PVDF as a binder and harmful solvent release was reduced during electrode processing. Activated carbon-carbon black composite electrodes with PEDOT as binder were found to have specific capacitance superior to that of activated carbon-carbon black electrodes with PVDF binder.

Keywords: Supercapacitor; EDLC, PEDOT:PSS; Activated carbon. 


\section{Introduction}

Supercapacitors, including electrochemical double layer capacitors (EDLCs) and redox or psedo-capacitors, are generally fast charging energy-storage devices with power and energy densities performance between traditional capacitors and batteries. They can be used as alternative or complementary power sources in various applications including telecommunication devices, standby power systems, portable electronic devices and electric hybrid vehicles [1]. An EDLC stores the energy by accumulating the opposite charges on the thin double layer $(5 \sim 10 \AA)$ [2] at the interface between the porous electrode and the electrolyte through electrostatic force. Carbonaceous materials such as aerogel [3][4] and powder [5][6] are the most commonly used electrode materials in EDLC devices due to carbon's high surface area, chemical and thermal stability, relatively low cost and low environmental impact. Carbonaceous materials are usually activated [4][7] to produce a large porous surface area before being used for EDLC electrodes. On the other hand, the activation of carbon results in a lower electrical conductivity, usually $0.1-1.0 \mathrm{Scm}^{-1}$, due to its high porosity and high surface area [8][9]. This large resistance inevitably results in an increase in power loss and energy dissipation. To improve the conductivity of carbon electrodes, conductive fillers, like carbon nanotubes (CNTs) [10][11] and carbon black [12] can be added. 5\% carbon black (CB) was mixed into AC to increase its conductivity [12] although high loading of $\mathrm{CB}$ in $\mathrm{AC}$ would induce a deterioration of the total capacitance due to the small surface area of the CB. 
A pseudo-capacitor stores energy through relatively fast and reversible faradaic (redox) processes in a redox-active material at characteristic potentials. Conducting polymers, such as polyacetylene, polypyrrole, polyaniline, polythiophene, and their derivatives, are amongst the more promising materials for a polymer-based pseudo-capacitor [13]. Conducting polymers are able to store charge, not only in the electrical double layer, but also throughout the body of the polymer by rapid faradaic charge transfer i.e a three-dimensional charge storage mechanism resulting in far higher capacity. However, the redox reaction in pseudo-capacitors is a slower process compared with the double layer process in EDLCs and, hence, the pseudo-capacitor has lower charge/discharge performance than an EDLC capacitor. One solution is to incorporate conducting polymers into the carbon electrode which has been shown to increase its capacitance while maintaining high level of power density [14][15].

Among conducting polymers, poly(ethylenedioxythiophene) (PEDOT) exhibits not only a high conductivity but also an unusual stability in the oxidized state, being considered as perhaps the most stable conducting polymer currently available [16] and, therefore, many researchers have studied PEDOT as the electrode material for supercapacitors [17][18][19].

PEDOT/carbon composite as electrodes for supercapacitors with high energy and power density have been reported [19][20]. But all the PEDOT reported in the PEDOT/carbon composite electrodes was synthesized in situ on carbon materials by 
electrochemical or chemical-polymerization from EDOT. It should be noted that although in situ synthesised PEDOT composite electrodes can have higher conductivity than the ones from mechanically blending of the ingredients, the increased process complexity and longer manufacturing times render this an unfeasible route for mass-production.

In this study, a PEDOT/carbon composite electrode was fabricated by mechanical blending with a low concentration of PEDOT, which also acted as a binder. The effect of PEDOT on the performance of carbon composite electrodes for supercapacitor devices was explored, where the carbon composite base was activated carbon (AC) and 5\% carbon black (CB). A commercial aqueous PSS doped PEDOT colloidal solution was used. The advantage of this aqueous PEDOT is that, after drying, the PEDOT/PSS film is highly conducting, mechanically durable, and insoluble in common solvents. When PEDOT was distributed in the AC matrix, it was found that the $\mathrm{AC}$ works as a scaffold, giving PEDOT a high surface-to-volume ratio, which improves both the energy and power density of the PEDOT itself through the increased surface area and by limiting the distance over which ion diffusion inside the PEDOT polymer body occurs. This aqueous PEDOT solution can work as a binder to replace conventional insulating binders such as PTFE and PVDF, reducing the resistance of the electrode and avoiding harmful solvents being released during electrode processing. The supercapacitor cells were assembled in a symmetric electrode configuration and $1 \mathrm{M}$ tetraethylammonium tetrafluoroborate $\left(\mathrm{Et}_{4} \mathrm{NBF}_{4}\right.$ or TEABF4) in propylene carbonate 
(PC) was used as electrolyte. $\mathrm{TEABF}_{4} / \mathrm{PC}$ electrolyte is widely used in batteries and capacitors as it allows a high electrochemical window of more than $3 \mathrm{~V} \mathrm{[1][21][22].} \mathrm{The}$ capacitor cells were subjected to electrochemical impedance spectroscopy (EIS), cyclic voltammetry (CV) and galvanostatic charge-discharge (GCD) tests. The data obtained were used to fit a generalised equivalent circuit model for each type of cell.

\section{Experimental details}

The composite electrodes were prepared from a blend of activated carbon (AC) (Sigma-Aldrich), Poly(3,4-ethylenedioxythiophene)-poly(styrenesulfonate) (PEDOT-PSS) (Sigma-Aldrich, 1.3wt\% dispersion in $\mathrm{H}_{2} \mathrm{O}$ ), Carbon black (acetylene) (Alfa Aesar) and Poly(vinylidene fluoride) (PVDF) (Sigma-Aldrich). All the chemicals were used without further treatment. Electrodes were constructed according to the ratios in Table $\mathbf{1}$ and were mixed thoroughly in PEDOT solution, or 1-methyl-2-pyrrolidinone (NMP) if PVDF was used, to form a slurry. The slurry was then coated onto aluminium foil using an Elcometer Film Applicator. The film was then dried in an oven at $200^{\circ} \mathrm{C}$ for 2 hours. The dried carbon film had a thickness of about $150 \mu \mathrm{m}$ and a loading of $\sim 5.5 \mathrm{mg} / \mathrm{cm}^{2}$. All the composite electrodes tested are listed in Table 1.

Table 1. Activated carbon composite electrodes for symmetric EDLCs

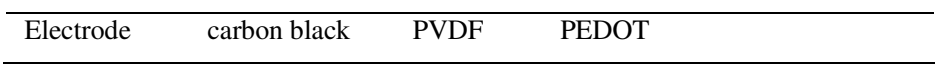




\begin{tabular}{lccc}
\hline & $(\mathrm{w} / \mathrm{w} \%)^{\mathrm{a}}$ & $(\mathrm{w} / \mathrm{w} \%)^{\mathrm{a}}$ & $(\mathrm{w} / \mathrm{w} \%)^{\mathrm{a}}$ \\
\hline ACB & 5 & 5 & 0 \\
ACBP5 & 5 & 0 & 5 \\
ACBP10 & 5 & 0 & 10 \\
\hline a weight percentage to the total carbon composite.
\end{tabular}

${ }^{\mathrm{a}}$ weight percentage to the total carbon composite.

The capacitor cells were assembled by placing two rectangular strips of carbon composite coated $\mathrm{Al}$ film with the carbon sides face to face. A piece of filter paper soaked with electrolyte solution was placed in between the strips as a separator. The electrodes overlap area defined the working area which was fixed to $2 \mathrm{~cm}^{2}$ in all the devices. Finally the device assembly was sealed in a plastic "bag" by vacuum.

The electrolyte was $1 \mathrm{M} \mathrm{Et}_{4} \mathrm{NBF}_{4}$ (Sigma-Aldrich, purity 99\%) in PC (Sigma-Aldrich, anhydrous, purity $99.7 \%)$.

The performances of different EDLC cells were characterized by EIS, CV, and GCD tests, using a VersaSTAT MC analyser. The devices were characterized in a 2-terminal configuration, no reference electrode was used in the tests.

\section{Results and discussion}




\subsection{Electrochemical impedance spectroscopy analysis and equivalent circuit}

models

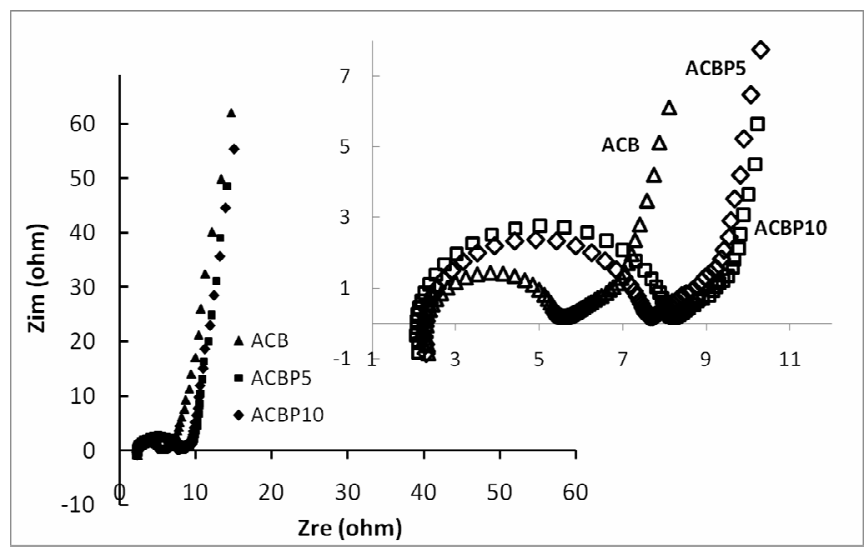

Fig 1. (a) Nyquist plots for the EDLC cells with ACB, ACBP5 and ACBP10 electrodes at a dc bias of $0.2 \mathrm{~V}$ and sinusoidal signal of $20 \mathrm{mV}$ over the frequency range $1000 \mathrm{kHz}$ - $0.01 \mathrm{~Hz} . Z_{r e}$ : real impedance. $Z_{i m}$ : imaginary impedance. Inset shows an enlarged scale of the plot.

The EIS measurements on the cells were carried out at a DC bias of $0.2 \mathrm{~V}$ with a sinusoidal signal of $20 \mathrm{mV}$ over the frequency range from $1 \mathrm{MHz}$ to $10 \mathrm{mHz}$. Fig 1 shows the corresponding Nyquist plots for the three devices with ACB, ACBP5 and ACBP10 electrodes. The Nyquist plots were used for the fitting of a generalised equivalent circuit model presented in Fig 2, consisting of an inductor L1 affecting the cell behaviour only at the highest frequencies, an equivalent series resistance R1, a parallel resistance $\mathrm{R} 2$, and three constant phase elements CPE1, CPE2, CPE3. A constant phase element (CPE) is described by the equation 
$z=\frac{1}{Q(\llbracket \omega) \rrbracket^{n}}$

For $\mathrm{n}=1$ the $\mathrm{CPE}$ is a capacitor with capacitance $\mathrm{Q}$. For $\mathrm{n}=0$ the $\mathrm{CPE}$ is a resistor with resistance 1/Q. Table 2 presents the values of the parameters of the generalised equivalent circuit model for each type of tested supercapacitor cell, where these parameters were derived from a best fit of the data of the corresponding Nyquist plots in Fig 1 using the EIS Spectrum Analyser ${ }^{\circledR}$ software. Fig 3 displays the Nyquist plots of the experimental data and the predictions of the corresponding equivalent circuit models using the parameters from Table 2.

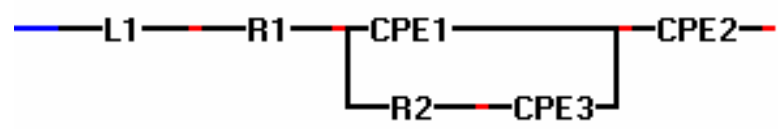

Fig 2. Generalised equivalent circuit model used for all supercapacitor cells in this study

Table 2. Parameters of the generalised equivalent circuit model for each type of cell (2 $\mathrm{cm}^{2}$ ) derived from the best numerical fitting of the experimental data of Nyquist plots 


\begin{tabular}{|l|l|l|l|l|l|l|l|l|l|}
\hline Cell with & L1 & R1 & CPE1: & CPE1: & R2 & CPE3: & CPE3: & CPE2: & CPE2: \\
electrodes: & $(\mu \mathrm{H})$ & $(\Omega)$ & Q1 & $\mathrm{n} 1$ & $(\Omega)$ & $\mathrm{Q} 3$ & $\mathrm{n} 3$ & $\mathrm{Q} 2$ & $\mathrm{n} 2$ \\
& & & $\left(\mathrm{Ss}^{\mathrm{n}}\right)$ & & & $\left(\mathrm{Ss}^{\mathrm{n}}\right)$ & & $\left(\mathrm{Ss}^{\mathrm{n}}\right)$ & \\
\hline ACB & 1.50 & 2.05 & $1.1 \times 10^{-3}$ & 0.87 & 3.1 & 1.10 & 0.1 & 1.59 & 0.947 \\
\hline ACBP5 & 1.35 & 2.05 & $3.1 \times 10^{-3}$ & 0.94 & 6.1 & 1.15 & 0.4 & 3.4 & 0.995 \\
\hline ACBP10 & 1.35 & 2.24 & $1.0 \times 10^{-3}$ & 0.93 & 5.35 & 1.10 & 0.3 & 2.35 & 0.956 \\
\hline
\end{tabular}
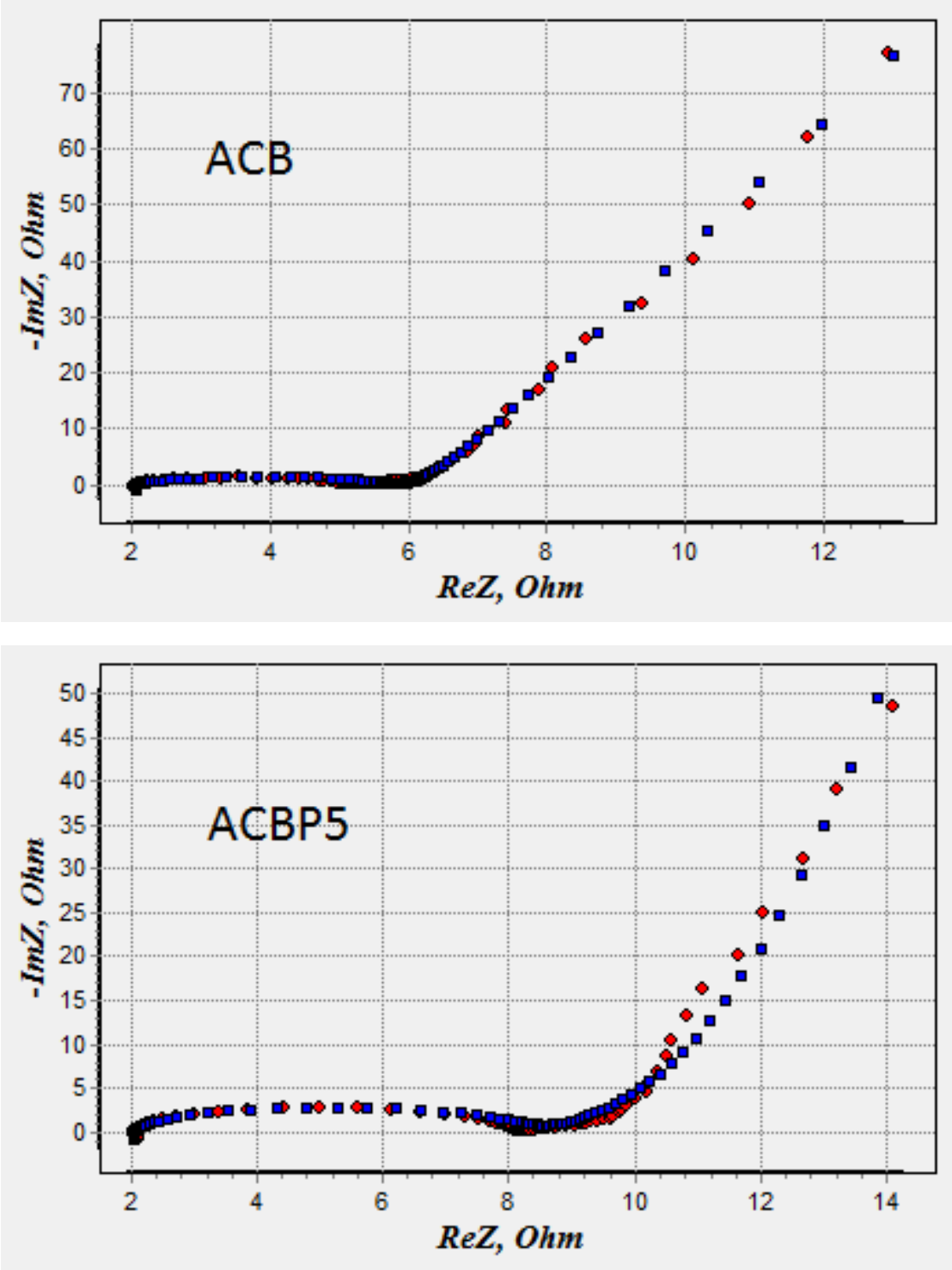


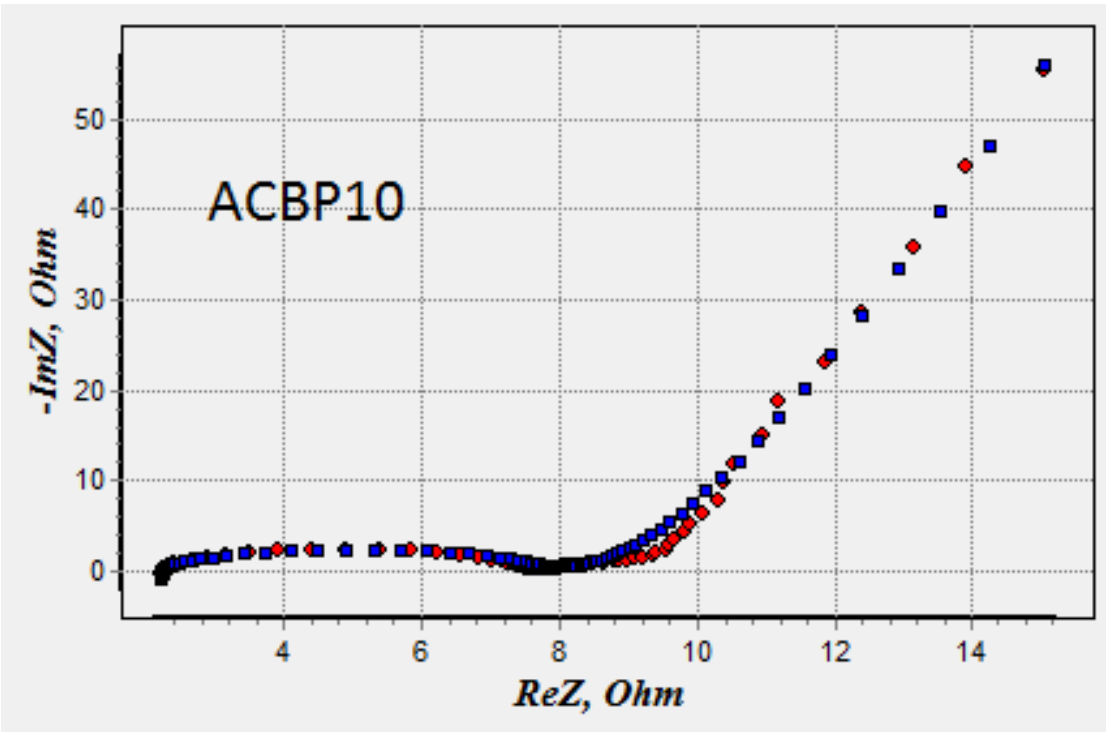

Fig 3. Experimental EIS data (red) and predictions (blue) of the generalised equivalent circuit model with parameter values displayed in Table 2

The first capacitor behaviour in EIS occurs in the region of high-to-medium frequencies illustrated by the semi-circle in the Nyquist plots in Fig $\mathbf{1}$ and $\mathbf{3}$ and represented by CPE1 in the generalized equivalent circuit model in Fig 2 . At these high-to-medium frequencies only small amounts of charge complexes in electrolyte can overcome the activation energy to migrate with the alternating potential, hence the capacitance is low developed by the moving electrolyte ions close to the Helmholtz plane represented by an interfacial contact capacitance $\left(C_{c}\right)$ and charge transfer resistance $\left(R_{c t}\right)$ within the micropores of porous carbon [23][24][25]. Lower $R_{c t}$ indicates better electrolyte pore accessibility. The closer the value of $n$ is to one in a $\mathrm{CPE}$ in the equivalent circuit model the closer to capacitor rather than resistor the element is. CPE1 has high n1 values for all cells (Table 2) indicating capacitor behavior, especially for the cells with PEDOT in the carbon composite electrode at 5 and $10 \%$ 
loadings. More specifically ACBP5 at 5\% PEDOT: loading has the highest CPE1 capacitance (semi-circle region).

The semicircle has two intersection points on the real axis: the first $Z_{r e}$ intersection point at the left end of the semicircle represents the ohmic resistance of the bulk electrolyte solution modeled by the equivalent-in-series resistance R1 in the equivalent circuit model in Fig 2, which is approximately the same for all three types of capacitor cells. The diameter of the semi-circle represents the charge transfer resistance, $R_{c t}$, modeled mainly by the in-parallel resistance R 2 in the equivalent circuit model: the results in Table 2 demonstrate that it is lowest in the case of ACB electrodes and it increases when PEDOT is added, indicating worse pore accessibility by the electrolyte in the latter case. This indicates that the addition of PEDOT has reduced the rate of charge transfer presumably by constricting part of the micro-pores of the $\mathrm{AC}$ and forming a higher resistance interface between the AC particles and the PEDOT. In the case of ACB which has PVDF binder, although PVDF is an insulator, when the electrode is dried at $200^{\circ} \mathrm{C}$ PVDF melts and shrinks through surface tension to expose AC micropores and to improve carbon particle-particle contact; as a result $R_{c t}$ is smaller in ACB than in the electrodes with PEDOT.

On the right side of the semicircle, the equivalent circuit model in Fig 2 includes a constant phase element CPE3, which in the case of ACB can be approximated by a resistor $(\mathrm{n} 3=0.1)$ that really adds to the value of the in-parallel resistance $\mathrm{R} 2$. In the case 
of ACBP5, containing 5\% PEDOT, CPE3 (n3 = 0.4) can be approximated by a $\sim 45^{\circ}$ Warburg element which represents the ion diffusion into the bulk of the electrode through pores of varying size [24]. Finally, at lower frequencies, the curve is almost vertical (Fig 1), indicating the capacitive nature of the device. This is represented by CPE2 in the equivalent circuit model, in which $\mathrm{n} 2 \approx 1$ for all types of cells in Table 2 indicating a good capacitor. Furthermore, the 5\% w/w PEDOT composition more than doubles the capacitance of CPE2 in comparison with that of the ACB based cell and in fact it seems to be the best PEDOT content for CPE2 to be the ideal capacitor (n2=1) and have the highest capacitance.

\subsection{Cyclic voltammetry}

In order to estimate the capacitance of the cells and stability of the electrolyte, cyclic voltametry $(\mathrm{CV})$ was carried out for the three types of cells in a series of sweeps at a rate from $0.01 \mathrm{~V} / \mathrm{s}$ to $1.0 \mathrm{~V} / \mathrm{s}$ between 0 and $3 \mathrm{~V}$. Fig 4 displays two typical $\mathrm{CV}$ curves at two extreme scan rates, where the specific capacitance of one electrode is given by the following equation:

$$
C=\frac{2 i}{m v}
$$
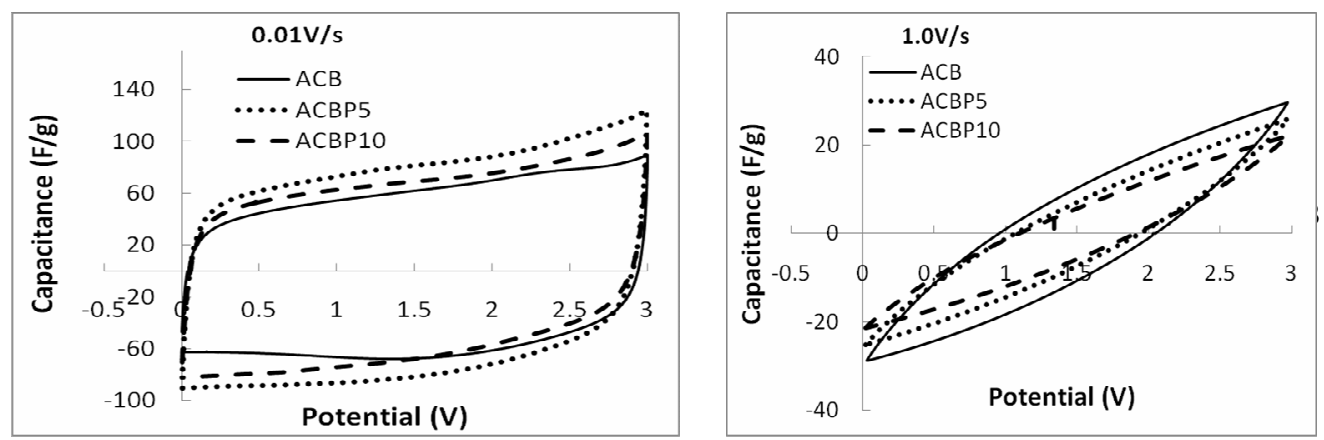
Fig 4. Cyclic voltammograms of EDLC cells with ACB, ACBP5 and ACBP10 electrodes using $1 \mathrm{M} \mathrm{TEABF}$ - $\mathrm{PC}$ electrolyte at two extreme sweep rates: (a) $0.01 \mathrm{~V} / \mathrm{s}$, (b) $1.0 \mathrm{~V} / \mathrm{s}$

where $i$ is the current, $v$ is the scan rate and $m$ is the mass of one. The curves are regular and reproducible, indicating good stability of the electrolyte in this operating voltage range. At low scan rate, all electrodes gave a $\mathrm{CV}$ shape closely resembling a rectangle. The capacitance value is in the order of ACBP5>ACBP10>ACB, indicating that addition of PEDOT in AC can increase the cell capacitance to a maximum and then decrease as the concentration of PEDOT increases, which is due to the pseudocapacitance contribution of PEDOT, limited by its resistance. Although the addition of PEDOT has increased the capacitance, no obvious redox peak of faradic contribution can be seen in the range of $0-3 \mathrm{~V}$; this does not mean that there is no redox reaction as conducting polymers contain a broad range of energy states, which can result in a wide redox peak [26]. In addition, as the CV was performed on the full EDLC cell, oxidation and reduction can happen at the positive and negative electrodes at different times which may have the effect of smoothing the redox peak of the full cell. 
At high sweep rates the CV curves of all electrodes were severely distorted to slim, leaf like shapes due to ions being able to only arrive at shallow positions within pores and the increasing diffusion resistance from the migration friction between the charge-complexes and the PC molecules, with the increasing sweep rate. The capacitance value is in the order of $\mathrm{ACB}>\mathrm{ACBP} 5>\mathrm{ACBP} 10$, due to the higher charge transfer resistance as the PEDOT concentration increases and due to the redox process being comparatively slow. Increasing the electrical conductivity of PEDOT might improve the situation.

\subsection{Galvanostatic charge-discharge tests}
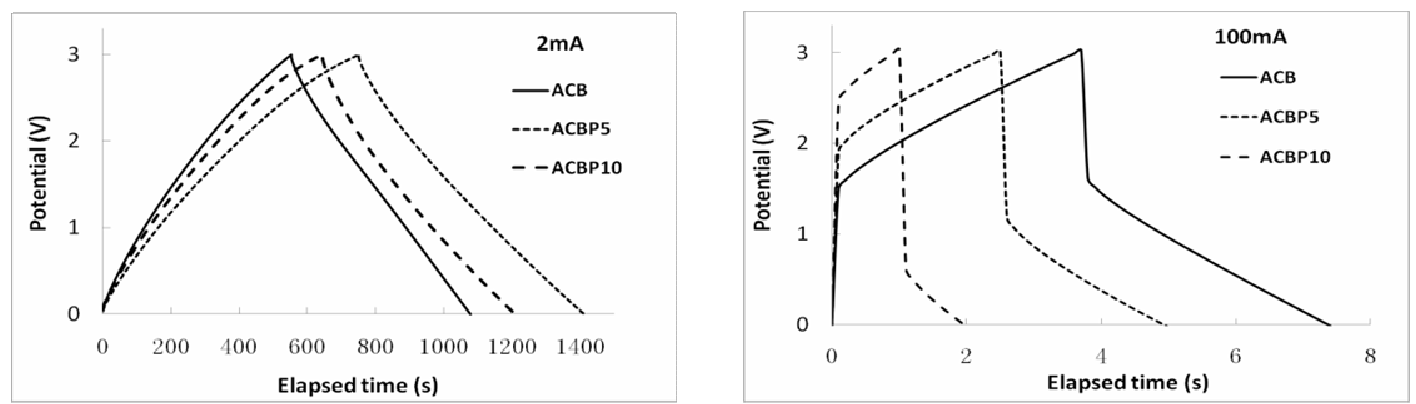

Fig 5. Galvanostatic charge-discharge curves of EDLC cells with ACB, ACBP2, ACBP5 and ACBP10 electrodes using $1 \mathrm{M} \mathrm{TEABF}_{4}-\mathrm{PC}$ electrolyte at two extreme constant currents: (a) $2 \mathrm{~mA}$, (b) $100 \mathrm{~mA}$. 
In order to examine the device performance including the dependence of the capacitance on the current density and evaluate the cell capacitance with respect to its power, charge-discharge cycling between 0 and $3 \mathrm{~V}$ at various currents from $2 \mathrm{~mA}$ to $100 \mathrm{~mA}$ was carried out. Fig 5 displays two typical charge-discharge plots at $1 \mathrm{~mA} / \mathrm{cm}^{2}$ and $50 \mathrm{~mA} / \mathrm{cm}^{2}$. At the low current density, $1 \mathrm{~mA} / \mathrm{cm}^{2}$ (or $2 \mathrm{~mA}$ current), all electrodes showed triangular charge-discharge curves, suggesting that the electrodes tested have no obvious redox reaction, which is consistent with the $\mathrm{CV}$ measurements. The performance (time elapsed during charge-discharge) of the three electrodes is in the order ACBP5>ACBP10> ACB, which is consistent with their CV behaviour at low scan rates. At the high current density of $50 \mathrm{~mA} / \mathrm{cm}^{2}$ (or $100 \mathrm{~mA}$ current), the performance of the electrodes is in the order $\mathrm{ACB}>\mathrm{ACBP} 5 \mathrm{ACBP} 10$, due to the increased internal resistance as the content of PEDOT increased. The $I R$ drop at the turning point of the charge-discharge line, which indicates the energy loss due to the internal or equivalent series resistance (ESR) [27], was enlarged at the higher current density. The $I R$ drop of the three electrodes is in the order ACB $<$ ACBP5 $<$ ACBP10, indicating that PEDOT increased the internal resistance, by coating the carbon particles. Although PEDOT is more conductive than PVDF, it does not shrink like PVDF at high temperature. However, PEDOT can be modified to increase its conductivity by adding a cosolvent such as DMSO [28].

The results of various $\mathrm{CV}$ and charge-discharge tests for the ACBP5 cell were 
assembled and converted to an energy density-power density Ragone plot for this single cell, which is presented in Fig 6. The plot illustrates that the addition of 5\% w/w PEDOT to the activated carbon-5\% w/w carbon black composite electrode increases the capacitance leading to increased energy density of the supercapacitor cell approaching $200 \mathrm{Wh} / \mathrm{kg}$ in our tests whereas the power density of the single small cell reaches a maximum value at $10 \mathrm{~kW} / \mathrm{kg}$.

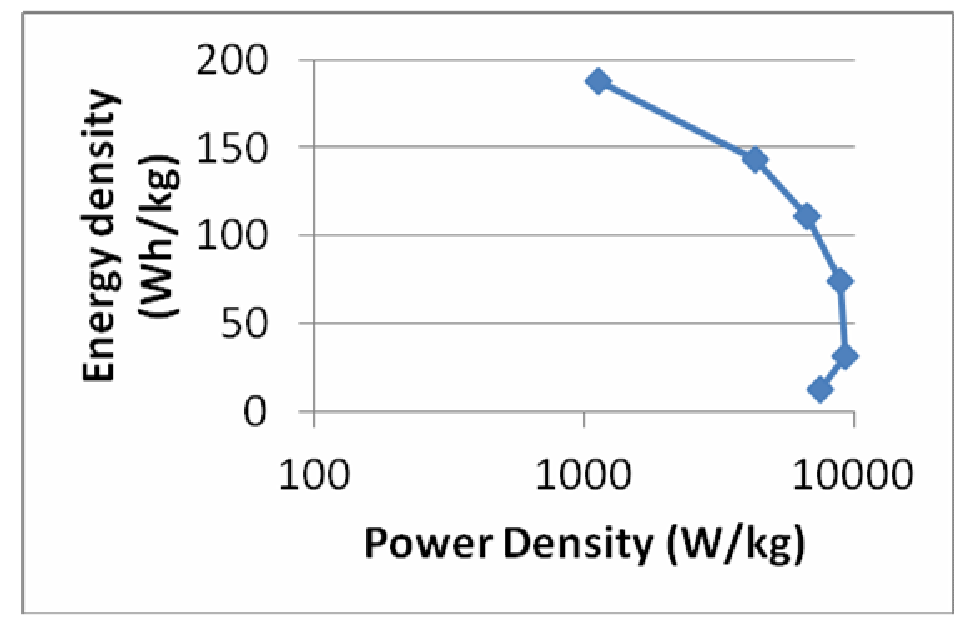

Fig 6. Ragone plot for the ACBP5 supercapacitor cell

\section{Conclusions}

This study has investigated the effect of PEDOT:PSS on an activated carbon-5\%w/w carbon black composite electrode used in supercapacitor cells with $1 \mathrm{M} \mathrm{TEABF}_{4}-\mathrm{PC}$ electrolyte. The fabricated cells were subjected to electrochemical impedance spectroscopy (EIS), cyclic voltametry and charge-discharge tests. A generalized equivalent circuit model with equivalent-in series-resistance, in-parallel resistance and three constant phase elements (CPEs) was fitted successfully to the EIS results for all 
types of cells.

In general, the addition of PEDOT:PSS enhanced the capacitance of the cell to a maximum at $5 \% \mathrm{w} / \mathrm{w}$ PEDOT, due to the additional pseudocapacitance of PEDOT. This is beneficial for capacitors used in low frequency applications, which still includes a large range of applications such as automotive energy storage. In fact, the capacitance of the CPE2 component of the equivalent circuit model was doubled by adding $5 \% \mathrm{w} / \mathrm{w}$ PEDOT, where CPE2 is the supercapacitor component functioning in the low frequency range. The work also demonstrated the feasibility of using PEDOT as a binder for AC which also reduces the release of harmful organic solvents during the electrode fabrication process. On the other hand, PEDOT contributed to a reduction in charge mobility and increase of the charge transfer resistance or the in parallel resistance in the equivalent circuit model.

\section{Acknowledgments}

This research was supported by the Engineering and Physical Sciences Research Council (EPSRC). We would like to thank Professor Joe Keddie in the Physics Department at the University of Surrey for the courtesy of facility usage in his laboratory.

\section{References}

[1] A. Burke, J. Power Sources 91(2000)37. 
[2] Y. Zhang, H. Feng, X. Wu, L. Wang, A. Zhang, T. Xia, H. Dong, X. Li, L. Zhang, Int. J. Hydrogen Energy 34(2009)4889

[3] J. Li, X. Wang, Q. Huang, S. Gamboa, P.J. Sebastian, J. Power Sources $158(2006) 784$.

[4] B. Fang, L. Binder, J. Power Sources 163(2006)616.

[5] V. Khomenko, E. Raymundo-Pĩnero, F. B’eguin, J. Power Sources 177(2008)643

[6] C.-C. Hu, C.-C. Wang, F.-C. Wu, R.-L. Tseng, Electrochim. Acta 52(2007)2498.

[7] H. Pröbstle, M. Wiener and J. Fricke, J. Porous Materials 10(2003)213.

[8] R. Chandrasekaran, Y. Soneda, J. Yamashita, M. Kodama, H. Hatori, J. Solid State Electrochem. 12(2008)1349.

[9] A.G. Pandolfo, A.F. Hollenkamp, J. Power Sources 157(2006)11.

[10] T. Bordjiba, M. Mohamedi, L.H. Dao, J. Power Sources 172(2007)991

[11] G. Lv, D. Wu, R. Fu, Z. Zhang, Z. Su, J. Non-Cryst. Solids 354(2008)4567

[12] Y.J. Kim, Y.A. Kim, T. Chino, H. Suezaki, M. Endo, M.S. Dresselhaus, Small 2(2006)339

[13] Graeme A. Snook, Pon Kao, Adam S. Best, Journal of Power Sources 196 (2011) 1.

[14] M. Mastragostino, R. Paraventi, and A. Zanelli: Supercapacitors based on composite polymer electrodes. J. Electrochem. Soc. 147(2000)3167.

[15] M. Selvakumar, D. Krishna Bhat, Journal of Applied Polymer Science, 107(2008)2165

[16] B. L. Groenendaal, F. Jonas, D. Freitag, H. Pielartzik and J. R. Reynolds, Adv. 
Mater., 12(2000)481.

[17] Kwang Sun Ryu, Young-Gi Lee, Young-Sik Hong, Yong Joon Park, Xianlan Wu, Kwang Man Kim, Man Gu Kang, Nam-Gyu Park, Soon Ho Chang, Electrochimica Acta 50 (2004) 843.

[18] Alexis Laforgue, Journal of Power Sources 196 (2011) 559.

[19] Ran Liu, Seung Il Cho and Sang Bok Lee, Nanotechnology 19 (2008) 215710.

[20] Timothy L. Kelly, Kazuhisa Yano, and Michael O. Wolf, ACS Appl. Mater.

Interfaces, 1(2009)2536

[21] A. Laheäär, H. Kurig, A. Jänes, E. Lust, Electrochim. Acta 54(2009)4587

[22] F.-Q. Li, Y.-Q. Lai, Z.-A. Zhang, H.-Q. Gao, J. Yang, Acta Phys. -Chim. Sin. 24(2008)1302

[23] K. Hung, C. Masarapu, T. Ko, B. Wei, J. Power Sources 193(2009)944.

[24] C. Masarapu, H.F. Zeng, K.H. Hung, and B. Wei, ACS Nano 3(2009)2199

[25] B. Fang and L. Binder, Electrochimica Acta 52(2007)6916.

[26] J. H. Chae, K. C. Ng and G. Z. Chen, Proc. IMechE Part A: J. Power and Energy 224(2010)479

[27] A. Izadi-Najafabadi, S. Yasuda, K. Kobashi, T. Yamada, D. N. Futaba, H. Hatori, M. Yumura, S. Iijima and K. Hata, Adv. Mater. 22(2010)E235.

[28] Hyo Joong Lee, Joowook Lee, and Su-Moon Park, J. Phys. Chem. B $114(2010) 2660$. 